Supporting Information

\title{
Photocurable 3D Printing Resin to Enable Laser-assisted Selective Electroless Metallization for Customized Electronics
}

Daegon Lee ${ }^{1}$, Bo-Young Kim ${ }^{1}$, Chan Ho Park², Gwajeong Jeong ${ }^{1}$, Seong-Dae Park ${ }^{1}$, Myong Jae Yoo ${ }^{1}$, Hyunseung Yang ${ }^{*}$, and Woo Sung Lee ${ }^{1^{*}}$

${ }^{1}$ : Electronic Convergence Materials \& Device Research Center, Korea Electronics Technology Institute, Gyeonggi-do, 13509, Republic of Korea

2 : Department of Electrical Engineering and Radiology, Stanford University, Stanford, CA 94305, USA

Keywords : 3D Printing, DLP, Metallization, Electroless Plating, ZnO

\section{Corresponding authors}

E-mail address:

hsyang@keti.re.kr (H. Yang)

wslee@keti.re.kr(W.S.Lee) 

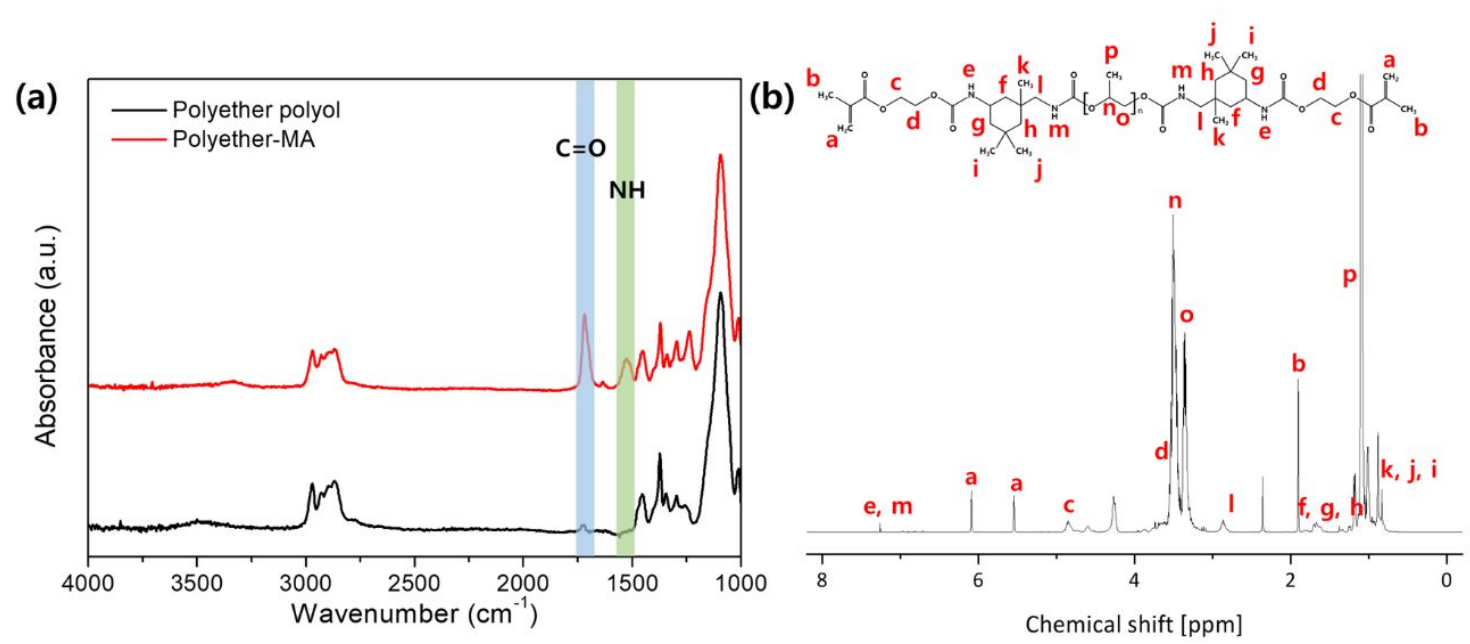

Figure S1. (a) FT-IR spectra and (b) ${ }^{1} \mathrm{H}-\mathrm{NMR}$ spectra of Polyether-MA

Table S1. SEC results of polyether polyol and Polyether-MA

\begin{tabular}{lccc}
\hline & $\mathbf{M}_{\mathbf{n}}(\mathbf{k g} / \mathbf{m o l})$ & $\mathbf{M}_{\mathbf{w}}(\mathrm{kg} / \mathbf{m o l})$ & PDI \\
\hline Polyether polyol & 2.4 & 2.6 & 1.03 \\
\hline Polyether-MA & 3.0 & 3.2 & 1.04 \\
\hline
\end{tabular}



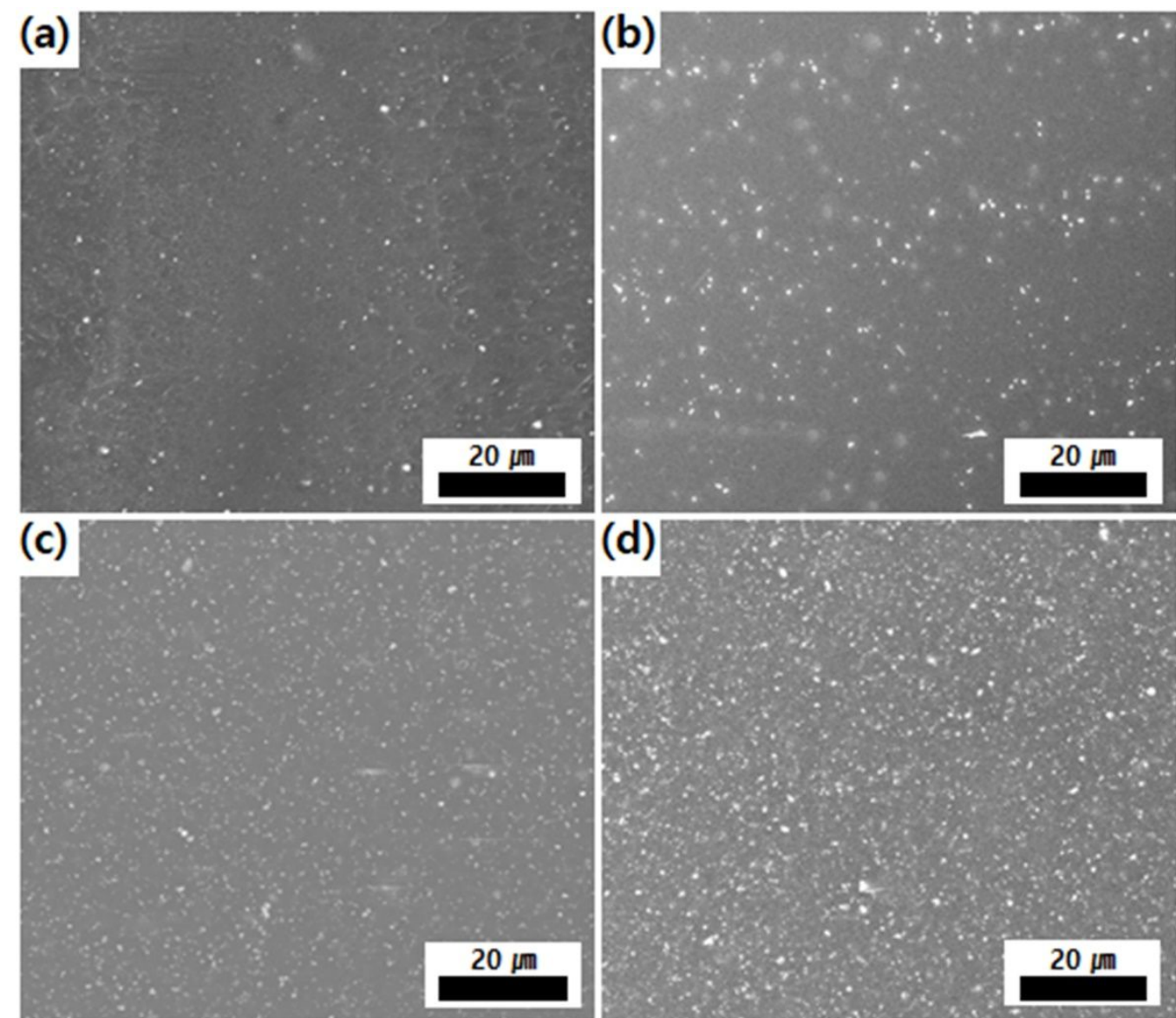

Figure S2. Cross-sectional SEM image of (a) 3DP-ZnO-1, (b) 3DP-ZnO-3, (c) 3DP-ZnO-5, and (d) 3DP-ZnO-9

Pristine ZnO NPs

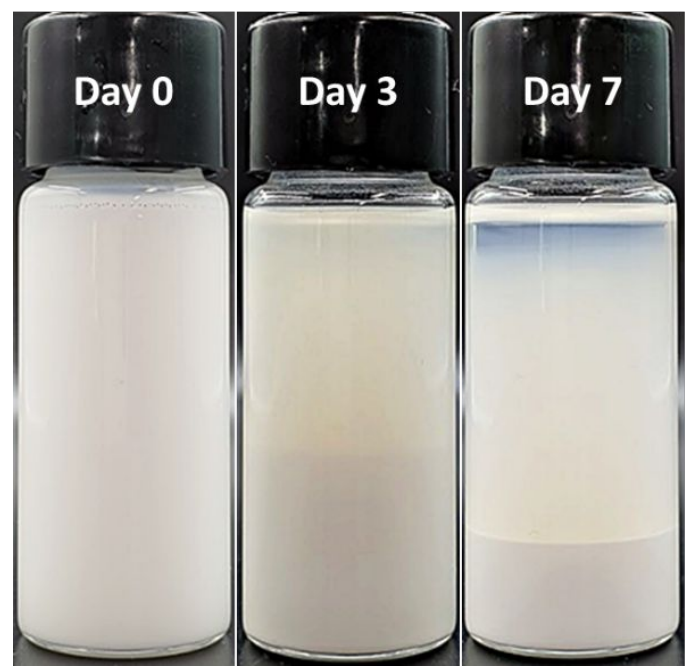

Figure S3. Image showing dispersion stability of 3DP-ZnO(P)-7 and 3DP-ZnO-7
Surface-modified ZnO NPs

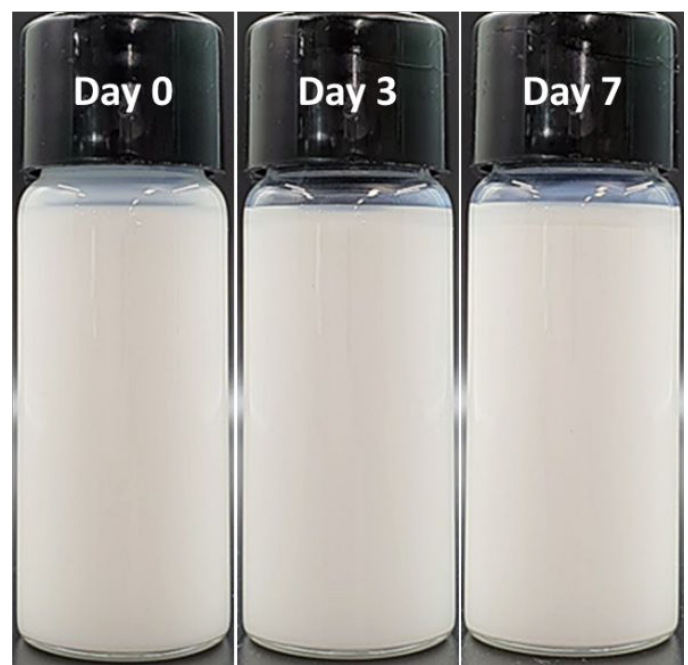

S-3 


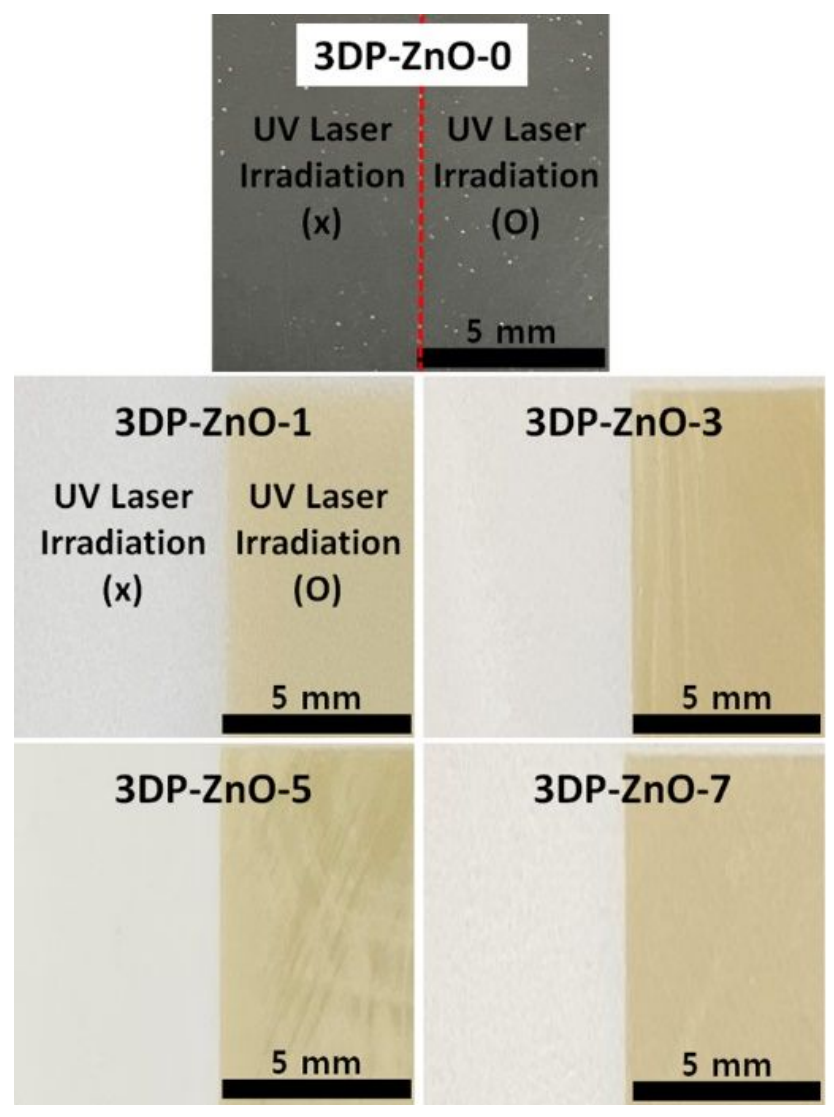

Figure S4. Photographic images of 3DP-ZnO before and after UV laser irradiation. 


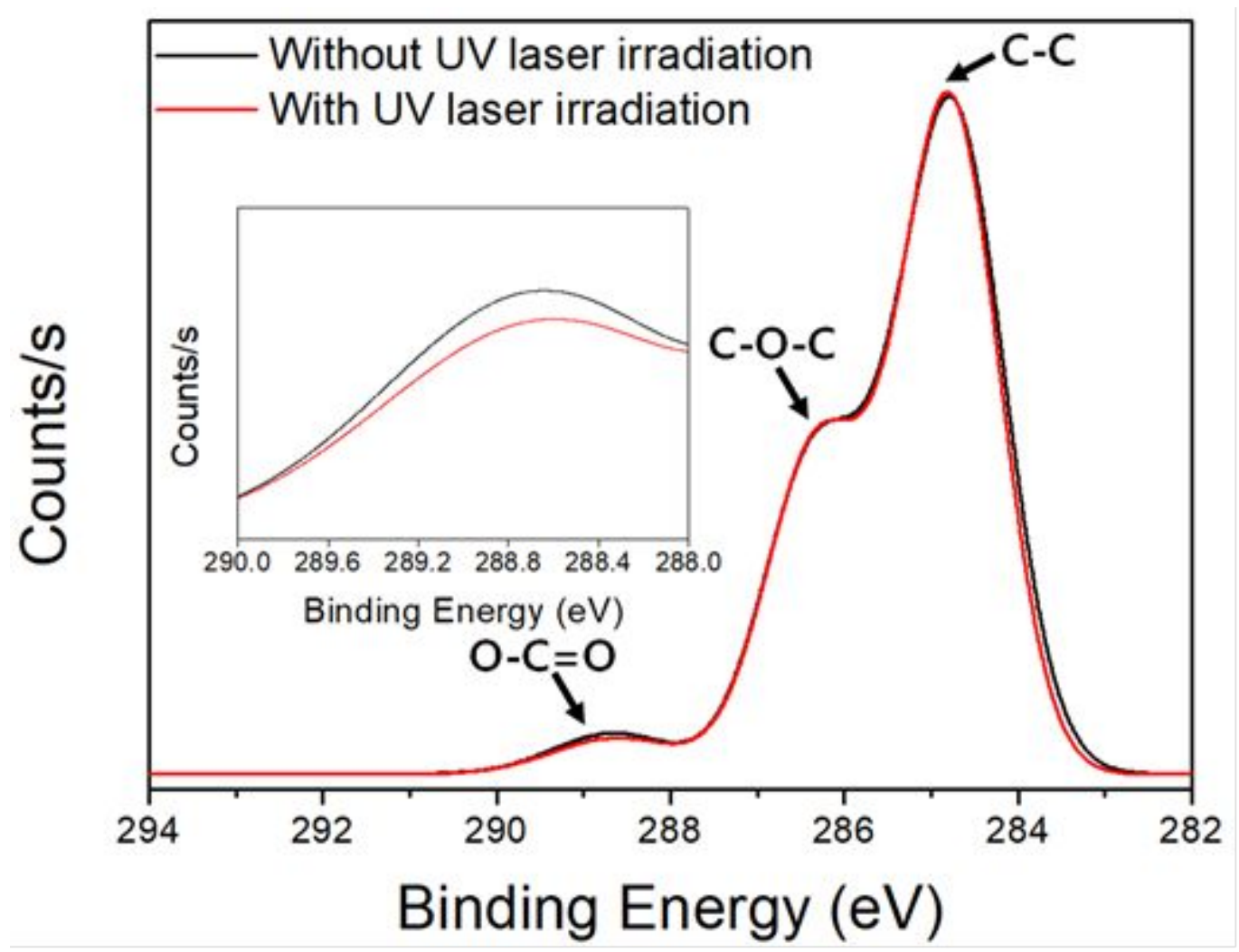

Figure S5. C 1s XPS spectra of 3DP-ZnO-7 before and after UV laser irradiation.

Table S2. Elemental composition of 3DP-ZnO7 before and after UV laser irradiation.

\begin{tabular}{cccccc}
\hline & C 1s & O 1s & N 1s & Zn 2p & C/O \\
\hline $\begin{array}{c}\text { 3DP-ZnO7 } \\
\text { before UV laser irradiation }\end{array}$ & 78.2 & 20.1 & 1.6 & 0.1 & 3.9 \\
\hline $\begin{array}{c}\text { 3DP-ZnO7 } \\
\text { after UV laser irradiation }\end{array}$ & 76.6 & 22.1 & 1.0 & 0.3 & 3.5 \\
\hline
\end{tabular}




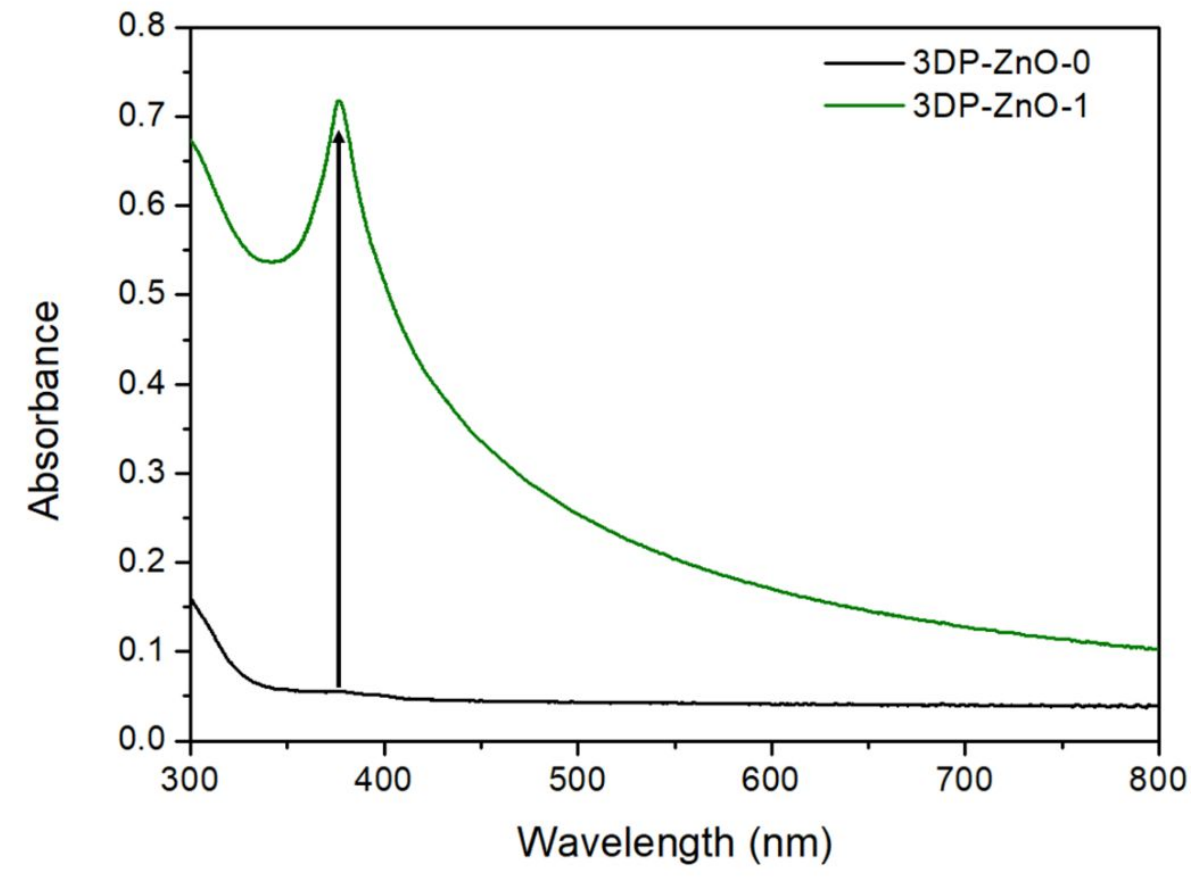

Figure S6. The absorption spectrum of 3DP-ZnO-0 and 3DP-ZnO-1

Table S3. The value of $\mathrm{R}_{\mathrm{a}}$ for a series of $3 \mathrm{DP}-\mathrm{ZnO}$ samples.

\begin{tabular}{ccccc}
\hline & 3DP-ZnO-1 & 3DP-ZnO-3 & 3DP-ZnO-5 & 3DP-ZnO-7 \\
\hline $\begin{array}{c}\text { before UV laser } \\
\text { irradiation }\end{array}$ & $0.16 \mu \mathrm{m}$ & $0.20 \mu \mathrm{m}$ & $0.21 \mu \mathrm{m}$ & $0.26 \mu \mathrm{m}$ \\
\hline $\begin{array}{c}\text { after UV laser } \\
\text { irradiation }\end{array}$ & $0.15 \mu \mathrm{m}$ & $0.41 \mu \mathrm{m}$ & $0.54 \mu \mathrm{m}$ & $1.26 \mu \mathrm{m}$ \\
\hline
\end{tabular}




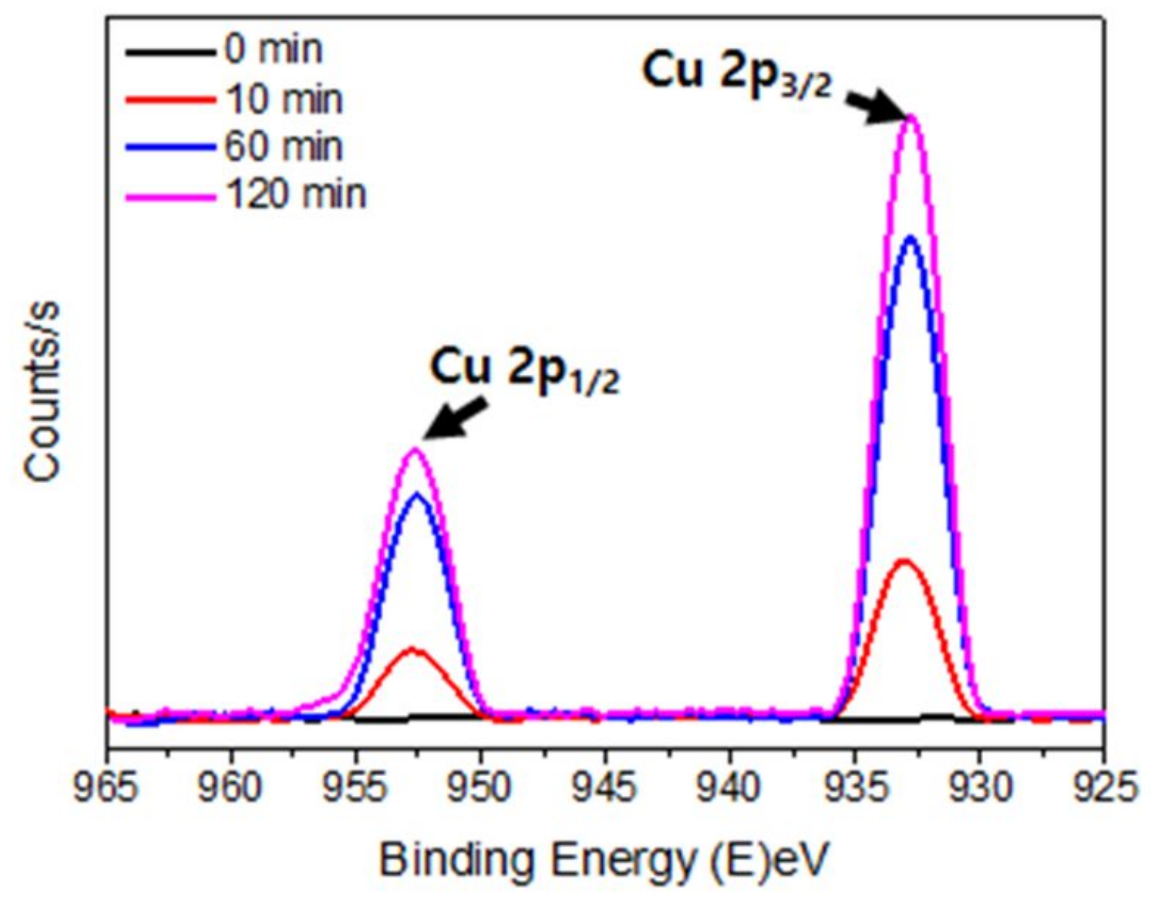

Figure S7. XPS spectra of $\mathrm{Cu} 2 \mathrm{p}$ for UV laser-irradiated 3DP-ZnO-7 as a function of $\mathrm{Cu}$ plating solution immersing time

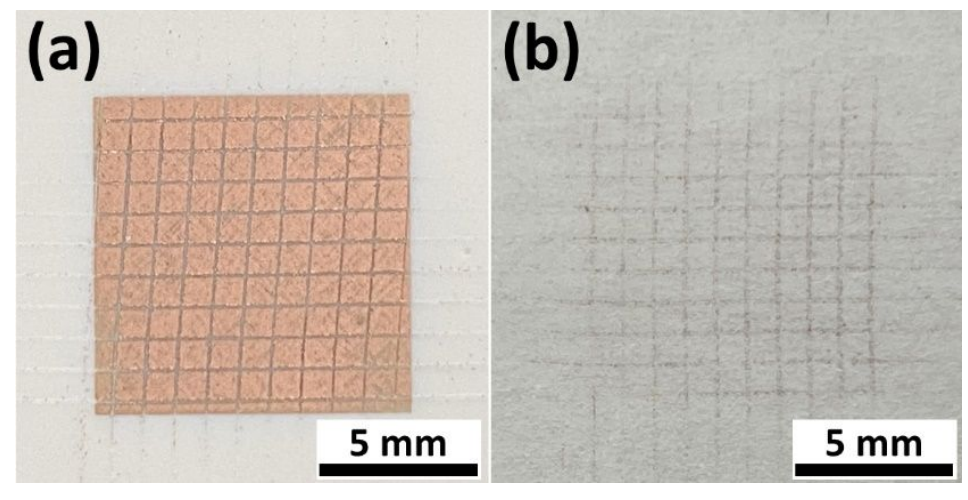

Figure S8. Photographic image of (a) Cu layers on 3DP-ZnO-7 and (b) transparent Scotch tape after cross hatch/scotch tape test 
Table S4. Electrical resistivity of $\mathrm{Cu}$ layers on 3DP-ZnO-7 after tape peeling test

\begin{tabular}{cccc}
\hline & Before tape peeling test & $\begin{array}{c}\text { After tape peeling test } \\
(\mathbf{1 s t})\end{array}$ & $\begin{array}{c}\text { After tape peeling } \\
\text { test (2nd) }\end{array}$ \\
\hline $\begin{array}{c}\text { Electrical } \\
\text { Resistivity } \\
\left(\times 10^{-6} \Omega \cdot \mathrm{cm}\right)\end{array}$ & $6.47 \pm 0.22$ & $6.44 \pm 2.05$ & $8.25 \pm 0.43$ \\
\hline
\end{tabular}

